\title{
¿QUIÉNES FUERON LOS VERDADEROS MOZÁRABES? UNA CONTRIBUCIÓN A LA HISTORIA DEL MOZARABISMO
}

En un artículo reciente, sostenía que nada justificaba el uso del término "mozárabe" para denominar a los cristianos que vivían en al-Andalus: "fueron mozárabes - decía en mi artículo - los que vivían en las regiones no musulmanas del norte de España..."'

Afirmaba entonces, y sigo afirmando, que aquellos cristianos tan implacablemente opuestos a la influencia del Islam, y ofendidos por la atracción que tenía el Islam en algunos sectores de la población indígena, no se llamaban, ni podría llamárseles, mozárabes. Esta palabra es sospechosa de cierta arabización, a la cual se oponía el grupo minoritario de clérigos, monjes y escritores de mediados del siglo IX, y contra la cual luchaban con todas sus fuerzas. Este grupo, al que pertenecían los mártires de Córdoba, mantenía celosamente sus conocimientos de literatura latina guardándolos como herencia preciosa de la época visigoda peninsular. Los escritos de estos cristianos, en particular los de San Eulogio y Âlvaro revelan una actitud religiosa inflexible, y una política antagónica a los musulmanes. Se advierte que los cristianos, quienes lamentaban cualquier señal de integración de los indígenas ${ }^{2}$, no hacían ningún esfuerzo por conseguir un ambiente de armonía.

En el siglo pasado, Simonet analizó profundamente el problema de los mártires en su apasionado. libro en defensa de los cristianos cordobeses: "Contrariada la cristianidad mozárabe en el

\footnotetext{
1 "El supuesto mozarabismo andaluz", Actas del I Congreso de historia de Andalucía, 1976, t. 1: Andalucía mediezal, Córdoba, 1978, pp. 149-151.

${ }^{2}$ Véase por ejemplo el argumento de Indiculus luminosus de Paulo Álvaro, y no simplemente la famosa queja de la arabización de algunos cristianos de estirpe patricia. Esta queja comprende el último párrafo de la obra y es de una interpretación dificilísima.
} 
sentimiento religioso, protestaban con el martirio"3. Quisiera señalar aquí la identificación explícita que hace Simonet de los cristianos cordobeses con lo mozárabe, identificación sostenida casi sin objeción en estudios posteriores. En el siglo XX, Cagigas habla de la "provocadora actitud de los mozárabes" y dice, refiriéndose a la muerte de San Eulogio, que "los mozárabes cristianos, como los patriotas cordobeses, habían perdido al animador infatigable de sus sueños de libertad"'. Aunque desde un punto de vista antagónico al de Simonet, Cagigas empleó el mismo vocabulario.

Entre los intérpretes actuales del episodio de los mártires, dos se han ocupado de examinar la cuestión terminológica ${ }^{5}$. Colbert reconoció el problema pero adoptó una postura ambivalente. Hizo observaciones acerca del significado de la palabra mozárabe, concluyendo que "las explicaciones basadas en una etimología árabe nada tienen que ver con los cristianos españoles que vivían bajo el imperio árabe en España"'. De acuerdo, pero Colbert estuvo lo suficientemente seguro como para afirmar que "la palabra mozárabe, cuando se la emplea en esta obra, significa los cristianos que vivían bajo el dominio musulmán en España". Así se entrevé la famosa "doble vertiente" de la palabra mozárabe en la frase de Chueca?. Hagerty, de la misma manera, ha hecho frente al problema sin encontrar una solución adecuada, aunque es consciente de que la palabra mozárabe es un término demasiado amplio, que no es útil porque incluye realidades muy distintas. Por una parte, serviría para describir a los arabizados como el obispo Rabīb Zayd del siglo $\mathrm{X}$, y al mismo tiempo a los cristianos no arabizados como San Eulogio y Pablo Alvar. Dice Hagerty que convendría utilizar el término ahl al-kitãb para referirse a los que estrecharon relaciones con los musulmanes; estos

${ }^{3}$ FRANCISco J. Simonet, Historia de los mozárabes de España, Madrid, 1903 (reimpresión: Amsterdam, 1967) especialmente los capítulos 12-21, pp. 319-502. Véase la cita en pp. 502-503.

${ }^{4}$ ISIDRO DE LAS CAGIGAS, Minorías étnico-religiosas de la Edad Media española, t. 1: Los mozárabes, Madrid, 1947, pp. 206 y 208.

${ }^{5}$ Dejo aparte aquí las obras de CARLETON M. SAGE, Faul Albar of Córdo ba: Studies on his life and writings, Washington, 1943, y NORMAN DANIEL, The Arabs and mediaeval Europe, London, 1975, cap. 2, porque no tratan el problema terminológico. Daniel llamó mozárabes a los principales protagonistas de los mártires cordobeses sin darse cuenta, al parecer, de la insuficiencia e incongruencia del empleo de la palabra mozárabe en este contexto.

${ }^{6}$ E. P. COLBERT, The martyrs of Córdoba (850-859): A study of the sources, Washington, 1962, pp. 22*23.

${ }^{7}$ FERNANDo CHUECA, Historia de la arquitectura española. Edad Antigua, Edad Media, Madrid, 1965, p. 129. 
pactados llegaron a aprender el árabe y por fin llegaron a ser "plenamente arabizados o sea mozárabes". Para no complicar las cosas demasiado, optó erróneamente, según mi parecer, por la palabra mozárabe. ¿Cómo contestar lo incontestable que propone? "Si una característica de los mozárabes es el uso de la lengua y costumbres árabes ¿qué decimos de San Eulogio y Paulo Âlvaro que tanto odiaban los islámicos?"' Hagerty insiste en que la palabra mozárabe "es un término de pura conveniencia ya que no tiene aplicación histórica alguna en [su] estudio"g. Tiene todo el derecho en decirlo y no me opongo a su opinión. El intento de justificar el uso de un término erróneo, aunque sea por pura "conveniencia práctica", confunde más que ayuda, porque los términos prácticos son muy ventajosos en la medida en que todo el mundo tenga pleno conocimiento de su significado. Declarar que "el ser mozárabe" es el final del proceso de arabización es reconocer el problema e intencionadamente desecharlo a la vez. Los mozárabes, el mozarabismo, la mozarabización tienen una amplitud que sobrepasa con mucho los confines de su tesis. Porfiar en el uso de "mozárabe" en contextos diferentes, según lo hace Hagerty, es un procedimiento a la vez inexacto e injustificado.

Algunos historiadores, en los últimos años, han tenido en cuenta la etimología de la palabra. Por ejemplo, O'Callaghan dice: "Cristianos, súbditos, de los musulmanes que adoptaron con el tiempo la lengua y costumbres árabes y llegaron a ser denominados mozárabes (mustarib, mustaribun), es decir como los árabes" ${ }^{\prime \prime}$. Se podría poner reparos a esta explicación: ¿por qué la primera documentación del término, en cualquiera de sus formas, en la Península ibérica consta en un documento latino leonés del primer tercio del siglo XI ${ }^{11}$ Más amplia y a la vez más prudente me parece la propuesta de Livermore, según la cual se debe limitar el empleo de la palabra mozárabe a un ambiente cristiano, es decir al territorio no islámico de la península ${ }^{12}$. Me gustaría presentar la situación en términos inequívocos. Los mo-

${ }^{8}$ Miguel J. HAGERTY, Los cuervos de San Vicente: escatología mozárabe, Madrid, 1978.

${ }^{9}$ HAGERTY, op. cit., p. 35.

${ }^{10}$ JOSEPH F. O'CALlaGHAN, $A$ history of medieval Spain, Corncll, 1975, p. 96. La traducción es mía.

${ }^{11}$ HITCHCOCK, art. cit., p. 150.

${ }^{12} \mathrm{H}$. V. LIVERMORE, The origins of Spain and Portugal, London, 1971, p. 376: "Mozarab is derived from mustarib, but it is not to be found before the year 1000, and its use has been avoided in this book. Evidently in alAndalus these people were simply Christians and their rite was the Hispano- 
zárabes fueron los que, emigrados de los pueblos del sur, sin preocuparse de convenciones religiosas, se establecieron en los reinos cristianos del norte. Creo que es preferible exponer el problema en tales términos, aunque reconozco la necesidad de sustentarlo cuidadosamente. Los historiadores de los mozárabes y del mozarabismo no podemos vivir siempre con un enigma intrínsecamente indescifrable. Me parece desconsiderado usar frases ambivalentes tales como "los mozárabes de ambos lados de las fronteras internas de la Península", especialmente cuando se reconoce que el problema es, al fin y al cabo, terminológico ${ }^{13}$.

En su libro citado, Cantarino somete el problema a un escrutinio detallado. Reconoce, o parece reconocer, una distinción entre el creyente de verdad y el creyente nominal, cuando, al referirse a Córdoba en el tiempo de los mártires, escribe: "con esta actitud de todo o nada llevada a sus últimas consecuencias, el cristianismo mozárabe renuncia a la creación de una auténtica cultura cristiano-árabe". Pero, ¿por qué incluir el calificativo mozárabe? A mi modo de ver, representa una equivocación fundamental emplear la palabra mozárabe en este contexto. Reconoce, pues, la intransigencia de los cristianos cordobeses, y para resolver el problema terminológico, propone una solución ingeniosa: "Si el nombre de mozárabe no fue introducido por los árabes para designar a los cristianos que vivían bajo su dominio, no es probable que fueran los cristianos del Norte los primeros en usarlo. Sería más lógico pensar, por el contrario, que fuesen los mismos mozárabes quienes lo introdujeran, en cuyo caso hubo de tener el significado, sin duda peyorativo, de colaborador o simpatizante sin referirse, al menos en principio, a todos los cristianos"14.

Según mi entender, todavía no se ha localizado un empleo de la palabra mustaribūn en una fuente hispanoárabe. Suponer que un mozárabe colaboraba con el adversario musulmán es idea atractiva a la vez que alucinante, pero, en mi opinión, el defecto fundamental de la hipótesis estriba en el hecho de que la palabra must ${ }^{c} a r i b a$ en su origen tenía una connotación de sectarismo.

Debemos considerar, en primer lugar, el sentido y la vivencia religiosa de la población indígena de al-Andalus. Cae fuera del argumento que intento presentar, que para los cristianos por vo-

Gothic rite. Only when they migrated to the north was it clear that they had become differentiated from northern Christians. The word Mozarab seems best restricted to this situación".

${ }^{13}$ VICENTE CANTARINO, Entre monjes y musulmanes. El conflicto que fue España, Madrid, 1978, p. 113.

${ }^{14}$ Cantarino, op. cit., pp. 108, 109. 
cación, los sacerdotes y los monjes, cuya existencia ha sido tan plenamente testimoniada por Simonet, la religiosidad era un aspecto fundamental de su vida. Sin embargo, estos cristianos, para quienes Leovigildo escribió su De habitu clericorum en el siglo IX, constituyeron una minoría en al-Andalus, permanecieron fieles a la cristiandad, resistieron la arabización en cuanto pudieron, y se aprovecharon de los privilegios concedidos al $a k l$ $a l$-kitã $b$, la gente del libro ${ }^{15}$.

La mayoría de la población indígena de al-Andalus no sentía ningún fervor religioso, y era gente desinteresada (si no apática) hacia la práctica de la religión fuera esta islámica o cristiana. Sostendría que, desde el principio del siglo VIII hasta, digamos, la fundación del califato omeya en el siglo x (año 929), por falta de cualquier indicación contraria en las fuentes primarias, existió una sociedad más bien irreligiosa, con la excepción arriba mencionada.

Poco a poco esta sociedad, especialmente dentro de las comunidades urbanas, se hizo más arabizada. Alguno que otro individuo pudo haberse convertido al islam, pero tales casos me parecen de importancia secundaria. Los indígenas adoptaron el idioma, las costumbres, los apellidos árabes por conveniencias prácticas y no por motivos religiosos, y en el siglo $\mathrm{X}$ esta gente arabizada constituyó la mayoría de la población de al-Andalus. No habían sido nunca ahl al-kitāa, ni beneficiarios de cualquier pacto. No eran nasāra, palabra reservada para los cristianos abiertos y activos, ni eran exactamente muwalladūn, término que se aplica normalmente, aunque no siempre acertadamente, a los que se habían convertido al islam. Tampoco se estima necesario postular que eran hijos de matrimonios mixtos para explicar su arabización. El problema terminológico se resuelve satisfactoriamente por la simple hipótesis de que estos indígenas fueron los auténticos must'ariba, pero debo advertir que no atribuyo ningún significado religioso a esta palabra. Must ariba es un sustantivo colectivo, que se refiere a cualquier comunidad caracterizada por su arabización, cuya religión, fuera ésta cristiana o islámica, no era factor predominante en su vida cotidiana.

No es mi intención dar la impresión de que era al-Andalus una comunidad carente de preocupaciones religiosas. Se practicaba el catolicismo pero había un reducido número de fieles ${ }^{16}$. Los

${ }^{15}$ F. J. SIMONET, "Los mozárabes de Córdoba", Ilustración Española y Americana, 2 (1897), 23-30.

${ }^{16}$ Estos cristianos han llamado la atención de los historiadores por la docu- 
laicos de la iglesia proseguían su culto sin la intervención, salvo raras excepciones, de las autoridades musulmanas. Su existencia era más bien mundana, con pocos motivos para inquietarse o para alegrarse a causa de las restricciones contra la práctica pública del cristianismo ${ }^{17}$. El islam se manifestaba más, pero en el corazón de la comunidad musulmana se encontraba en las familias árabes de las ciudades principales que ejercían el poderío. En cuanto disminuyó la autoridad de estas familias, en particular durante los años calamitosos que precedieron a la disolución del califato en el año 1031, disminuyó la vitalidad del Islam. Hacia fines del siglo $\mathrm{XI}$, cuando las familias hispanoárabes se entregaban a luchas fratricidas, los juristas malikíes llamaron a los almorávides magrebinos, para restaurar los valores vitales del Islam.

En resumen, cabe reiterar que, aunque existían musulmanes y cristianos piadosos, su número era reducido. La mayor parte de la comunidad se inclinó con el tiempo a la lengua y las costumbres árabes, motivada más bien por conveniencia y provecho que por impulsos espirituales. Estos arabizados se adaptaban al Islam en caso de necesidad, se abstenían del comercio durante las horas de la oración semanal, se guardaban de defender a las autoridades religiosas y jurídicas.

Prestemos atención ahora al fenómeno mozárabe en lo que considero su verdadero contexto, el territorio no islámico del norte de la Península. La aparición más temprana del derivado mozárabe en cualquier variante en la Península ibérica, se encuentra en un documento del año 1026, perteneciente al archivo de un monasterio leonés, San Cipriano de Valdesalce. La palabra aparece en otro documento leonés, sin fecha, pero del mismo siglo $\mathrm{XI}^{18}$. No he encontrado más ejemplos del término en territorio leonés, así que, al parecer, estos dos casos son aislados. Después de la conquista de Toledo, en el año 1085, comienza a aparecer con frecuencia como apellido entre la gente arabizada que habitaban tierras toledanas ${ }^{19}$; y en los siglos posteriores, varios fueros municipales hacen mención de mozárabes, con fre-

mentación que se conserva, pero en cuanto a la cuestión que nos ocupa son de escasa importancia.

${ }^{17}$ Véanse por ejemplo los datos presentados por al Jušní en JULIẤN RIBERA, Historia de los jueces de Córdoba por Aljoxani, Madrid, 1914.

${ }^{18}$ Doy el texto de ambos documentos, junto con un estudio del contenido en el cuarto capitulo de mi tesis doctoral: An examination of the use of the term "mozarab" in eleventh and twelfth-century Spain, St. Andrews, 1971. Véase también Hitchcock, art. cit., p. 151.

${ }^{19}$ Hay amplia información de la arabización de la comunidad en A. GON- 
cuencia como beneficiarios de una legislación privilegiada ${ }^{20}$. A base de esta evidencia terminológica, es claro que la palabra mozárabe se estableció en la península en el siglo XII, probablemente en ambiente toledano.

Por todo lo expuesto, quizás parezca paradójico afirmar que el mozarabismo - la diseminación de influencias islámicas dentro de una comunidad cristiana - se originó en territorios leoneses. Es precisamente porque se manifiestan semejantes características en tierras toledanas un siglo más tarde que se puede emplear el mismo vocabulario. Aunque la mozarabía de Toledo ha sido objeto de varios estudios, el mozarabismo leonés, tema amplio, sigue sin la atención que, por su importancia, merece ${ }^{21}$. Para nuestro propósito es de suma importancia, por lo tanto, tratar de evaluar todas las pruebas disponibles. Es muy conocida la abundancia de palabras y nombres árabes en los documentos latinos redactados en los monasterios cristianos de los territorios de León y Castilla en los siglos X y XI. No me ocuparé aquí del conjunto abigarrado de palabras que se refieren a prendas de lujo o de otro tipo, sedas, bordados y lo que llama Gómez Moreno "preseas eclesiásticas"22. Me limitaré a los nombres propios de origen árabe que proliferan en los documentos, y me atrevería a afirmar que estos nombres, casi todos, pertenecen a aquel elemento heterogéneo de alAndalus que he designado must ariba, es decir neutrales en cuanto a sus lealtades religiosas. A esta gente se debe la propagación del mozarabismo dentro del territorio leonés.

Cabe pensar en una situación propicia para los inmigrantes de al-Andalus en la segunda mitad del siglo IX y durante el siglo

ZÁlez PAlencia, Los mozárabes de Toledo en los siglos xii y xiii, 4 ts., Madrid, 1926-1930.

${ }^{20}$ Trato el tema en el cap. 5 de mi tesis citada supra, nota 18.

${ }^{21}$ Aparte de las fuentes primarias, publicadas por GONZÁlEZ PALENCIA, op. cit. supra, nota 19, véanse los estudios de REYNA PASTOR DE TOGNERI, Conflictos sociales y estancamiento económico en la España medieval, Barcelona, 1973, y Del Islam al cristianismo en las fronteras de dos formaciones económico-sociales, Barcelona, 1975. En cuanto a León, hay que recurrir, para los textos primarios, a los libros de MANUEL GÓMEZ MORENo, Iglesias mozárabes, Madrid, 1919 (reimpr. Granada, 1975), y de CLAUDIO SÁNCHEZ AlboRnoz, Una ciudad de la España cristiana hace mil años, $5^{a}$ ed., Madrid,.1966, y sus estudios más recientes.

${ }^{22}$ Véase, entre otros, Gómez MORENo, op. cit., cap. 10; A. STEIGER, Contribución a la fonética del hispano-árabe y de los arabismos en el iberoromano y el siciliano, Madrid, 1932; id., "Zur sprache der Mozaraber", Festschrift Jacob Jud, Romanica Helvetica, 20 (1943), 624-723; id., "Un inventario mozárabe de la iglesia de Covarrubias", AlAn, 21 (1956), 93-109; y C. E. DUBLER, sobre el libro de Steiger, AlAn, 9 (1944), 256-261. 
$\mathrm{X}$, épocas en que se ofrecieron condiciones favorables a comunidades de monjes que querían establecerse en tierras incultas para explotarlas. Según una inscripción, el abad Juan, procedente de Córdoba, restauró en cinco meses, en el año 921, el monasterio de San Martín de Castañeda, Astorga, junto con los monjes de su séquito $^{23}$. El monasterio de San Miguel de Escalada estaba en ruinas cuando llegó, desde Córdoba, el abad Alfonso con sus compañeros; ellos lograron reedificarlo en menos de doce meses y el obispo de León lo consagró el 20 de noviembre de 913. Podríamos multiplicar ejemplos como éstos. Los nombres cristianos de los abades mencionados y de otros más indican que fueron cristianos, verdaderos creyentes, quienes se marcharon de alAndalus por motivos no especificados, quizás para aprovechar como los demás inmigrantes al territorio leonés, el programa de colonización puesto en vigor por los monarcas, tales como Alfonso III de Asturias y de León. Los monasterios prosperaron y aumentaron rápidamente su influencia y su riqueza durante siglo y medio hasta la divulgación de las reformas cluniacenses al sur de los Pirineos. Pero los colonizadores trajeron consigo sus propias artes y artesanos: tejedores, albañiles, carpinteros, herreros, vidrieros; algunos se asociaron con las comunidades monásticas, otros se instalaron en aldeas y pueblos. Todos practicaron sus oficios como lo habían hecho en al-Andalus, contribuyendo así al influjo de una forma de vida arabizada en toda la región leonesa. La naturaleza de las comunidades que evolucionaron se pareció fielmente a la de su vida anterior. El siglo X marcó así la aparición de la época propiamente denominada mozárabe. Los responsables de la propagación de este nuevo estilo de vida eran arabizados, no cristianos apasionados por la preservación de una herencia latinovisigoda, ni bereberes ni musulmanes en busca de prosélitos y con el propósito de dejar huellas imborrables en tierras leonesas. Se han propuesto diversas teorías ${ }^{24}$, pero creo que esos migrantes eran hombres y mujeres ordinarios que habían vivido anteriormente en un ambiente arabizado y que ahora, en otro territorio, bajo otro dominio, perpetuaban tranquilamente el estilo y nivel de vida que les eran familiares. Esta gente llevaba nombres árabes, lo que era totalmente nor-

${ }^{23}$ Los textos de las inscripciones están reproducidos en E. HÜBNER, Inscriptiones Hispanicae Christianae, Pars Secunda-Baetica, Berlín 1871, p. 87, núm. 275 y en el suplemento (Berlín, 1900), p. 107, núm. 469.

${ }^{24}$ GómeZ MORENo, op. cit., pp. 116-118, resume estas teorías. Dice, en p. 116, que "aquellas gentes arabizadas leonesas. . . eran cristianas en su totalidad". 
mal, aunque había excepciones, desde luego, como en el caso de los esclavos ${ }^{25}$. Sin embargo, repito, en los documentos leoneses de los siglos X y XI, los nombres árabes pertenecían, en su vasta mayoría, a inmigrados voluntarios, gente arabizada que no observaba ninguna religión en particular.

Pues bien, el inventario de nombres recogido por Gómez Moreno en sus Iglesias mozárabes plantea problemas que no tienen fácil solución. Conviene, en primer lugar, no perder de vista que esos nombres representan una pequeña porción de la onomástica árabe que figura en todos los cartularios, tumbos, becerros y archivos monásticos existentes. La lista recopilada por Gómez Moreno puede ser una fracción infinitesimal del total de tales nombres. Me limito al ejemplo del becerro de Celanova, el cual comprende documentos del siglo $\mathrm{X}$ y de la primera mitad del siglo XI. El becerro consta de doscientos folios en los cuales se encuentran más de cuatrocientos nombres árabes identificables. Algunos, como el presbítero Homari, son clérigos ${ }^{26}$, pero la mayor parte de ellos son laicos, testigos de documentos de compra y venta, de donaciones o de testamentos. Abundan signatarios árabes cuyo oficio es imposible identificar. Los tumbos legionense y astoricense, que incluyen documentos pertenecientes a una cantidad de monasterios de menor prestigio, cuentan la misma historia.

Para demostrar mi teoría he seleccionado la lista de los nombres árabes de "dieciocho abades", recopilada por Gómez More$\mathrm{no}^{27}$. Elijo esta lista porque, aunque parece que contradice todo lo expuesto arriba, en realidad lo confirma. Estos abades con nombres árabes se diferencian de aquéllos llamados Alfonso, Juan, y Martín, porque, si hubieran sido cristianos del sur, hubieran comprometido su posición por no tener nombres claramente distintos de sus adversarios. Hay que recordar aquí el caso de San Eulogio y de los otros mártires. Soy partidario de la hipó-

${ }^{25}$ Véase el interesante estudio de EDUARDo HINOJOSA, Documentos para la historia de las instituciones de León y de Castilla (siglos $x$-xiii), Madrid, 1919, especialmente el documento 28.

${ }^{26}$ Becerro de Celanova, fol. 42v, documento con fecha del año 905.

${ }^{27}$ Los abades son, según Gómez Moreno, "Domno Melic; Abeiza; Zanom; Abogalebh presbiter; Domno Vellite de Valdevimen; Ramellus de Algadef; Domno Hisccam de Valcavato; Hilal; Zithaius o Citaius de Apeliare; Salvatus cognomento Hilal de Valdesálice; Domno Eiza de Algadef; Daudi y Citi en el Bierzo; Theodomiro cognomento Muza, de San Dictino, en Astorga; Havive y Citte; Habibi, de San Prudencio, en la Rioja; y Petrus prolix Citis, o Petro Citiz, de Sta. Marta de Tera". Además nombra a dos abadesas "Hamama y Habba cognomento Leokadia, en Santiago de León”, op. cit., p. 108, notas 5 y 6 . 
tesis siguiente: si la vocación cristiana se revelara explícitamente por ciertos detalles como el añadido de abad, diácono, presbí. tero, o monje a un nombre árabe, esto indicaría su asociación con el cristianismo en el lugar de arraigo, pero no necesariamente que los individuos eran cristianos activos en al-Andalus. Puesto que ostentaban nombres árabes, el supuesto me parece dudoso. Contra lo que afirma Gómez Moreno, encuentro muy posible que una "carrera en la iglesia" fuera atractiva para algunos pobladores recién llegados del sur; no hay inconveniente en suponer que hubieran adoptado el cristianismo, a pesar de su arabización, sin dar ninguna importancia a los nombres que tenían.

Queda todavía por tratar el fenómeno de los abades con nombres árabes. Algunos nombres, tales como Ramellus y Daudi, no deben figurar en la lista, porque no son de origen árabe; además, Citi y sus derivados constituyen un caso incierto. Raras veces se utilizaba Sayyid o Sid como nombre propio, ya que era más bien un título para el dueño de la hacienda, o quizás para el superior eclesiástico. Aunque el uso de la palabra abunda en los documentos, no es un indicio infalible del origen árabe de estos individuos. Hay que eliminar tres nombres más: Theodomiro, cognomento Muza, Citte y Havive porque la lectura de 'abba' no se justifica ${ }^{28}$. Entre el núcleo de unos diez nombres que quedan en la lista, me limitaré a mencionar dos: Zanom y Hilal.

Zanom o Zanon, si es nombre árabe, es muy poco común, quizás caso único ${ }^{29}$. Con sus compañeros, cuyos nombres descubren plenamente su origen árabe, Zanon edificó el monasterio de Peñalba (Pinnae Alba) ${ }^{30}$, bajo la dirección del obispo Salomón de Astorga. Le ayudaban entre otros, Iben Zaute es decir Ibn Sa'üd, Habdela Ibenaunar ('Abdulla b. 'Umar), Didacus

${ }^{28}$ Theodomirus cognomento Musa Alba, documento del año 1014 (ms. 9194, B. N. M., fol. 77v); Citte Alva, Havive Alva, documento del año 1029 (ms. 9194, fol. 86v). Figuran en este grupo de documentos varios Albas, tales como Vimara Alva y Belge Alva en un documento del año 992 (fol. 74), todo lo que hace sospechar de un nombre propio. Además, hay un Zamai de Alva en un documento del año 1058, y un Zamayel equinomitus (es decir economicus) fue testigo de una donación en favor de iglesias astoricenses en el año 1063. Si es el mismo hombre, como lo es con toda probabilidad, ¿habría cambiado de profesión? Hay más casos parecidos.

${ }^{29}$ Zanon Abba testis, documento de 937 (ms. 1197B del Archivo Histórico Nacional, Madrid, fol. 214), y España Sagrada 16, p. 438 donde se reproduce este documento; Zanon abba en un documento de 939 (ms. 9194 de la B. N. M., fol. 70); y un Zanon notarius, testigo de un documento del año 940 (ms. 1197B del Archivo Histórico. Nacional, fol. 197).

${ }^{30}$ Para éste, véase Gómez MORENo, op. cit., pp. 224-225. 
Iben Froila, dos nombres visigodos unidos por la palabra árabe "hijo" y Âlvaro Ibnzalem, híbrido visigodo-árabe. El nombre Ālvaro sugiere que el matrimonio decidió dar a su hijo un nombre adecuado al nuevo ambiente leonés. El nombre Ibn Salim se adecuaba bien al ambiente islámico, pero no era apropiado para el hijo en circunstancias diferentes. Hay que señalar, sin embargo, que los nombres, al parecer, no provocaban reacciones negativas en los reinos cristianos. Estos testimonios nos dicen, ni más ni menos, que había inmigrados para quienes la necesidad de adoptar nombres menos comprometedores no era urgente todavía. Poco a poco los nombres se acomodaban al sistema empleado en los territorios norteños de la Península, y no es marcada la propensión a conservar aquellos que señalaban sus lugares de procedencia. El empleo de tales nombres perduraba normalmente una generación, y, en adelante, a veces a través de formas híbridas, desaparecía.

Hilal es nombre árabe familiar, aunque podría confundírselo de vez en cuando con Hilarius. Este Hilal fue el primer abad del monasterio de San Cipriano de Valdesalce, cerca de Coyanza, hoy pueblo de Valencia de Don Juan, provincia de León desde el año 974 hasta el año 1000. Consta, sin lugar de dudas, en seis documentos registrados en el Tumbo legionense. En los tres casos más tempranos se lee Hilale Abba o Domno Hilale Abba, en el cuarto Hilalius Abba, en el quinto Salvatus abba cognomento Hilal, y en el sexto Salvatus abba et confessus cognomento Hilal ${ }^{31}$.

Hace muchos años, Díaz Jiménez se refirió en una nota al monasterio de San Cipriano de Valdesalce, única ocasión en que dicho monasterio ha sido digno de una mención por los historiadores $^{32}$. Díaz Jiménez habla de un documento del 20 de octubre de 992, que, desafortunadamente, no he podido localizar. Al parecer, este documento menciona un incidente en que el presbí-

${ }^{81}$ Tumbo legionense, fols. 173v-174; 174; 164-164v; 176; 167-167v, este documento está reproducido en España Sagrada, 36, ap. I, I-II; y fols. 168 169, el testamento de Hilal, reproducido en ibid., ap. III, IV-VI.

${ }^{32}$ ELOY DIAZ JIMÉNEZ Y MOLLEDA, "Datos para la historia del monasterio de San Justo y Pastor", $H M P, 3,165-169$. Los apuntes están en las páginas 167-168. Algunos de los datos de Díaz Jiménez son inexactos, y estas inexactitudes se repiten en el artículo de la Enciclopedia universal ilustrada, Madrid, 1958 , t. 66 , p. 521, que cita el artículo de Díaz Jiménez como fuente bibliográfica única. Doy el resumen de sesenta y cinco documentos recogidos del tumbo legionense en mi tesis doctoral, precedido de un estudio y con índices onomástico y toponímico, apéndice II, pp. 135-187. Dejo para otra ocasión un examen detenido de este monasterio. 
tero Julián se negó a dotar un monasterio porque su abad había cometido un pecado escandaloso en León. Según Díaz Jiménez ese documento especifica que el monasterio aludido fue Valdesalce y que fue Hilal, nombrado Salvatus, quien pecó. Como castigo a la ofensa, cualquiera que haya sido, se impuso a Salvatus una multa de doscientos sueldos. En la última disposición de sus bienes, Hilal se califica a sí mismo "confessus" en una fórmula curiosa "Salvatus abba et confessus cognomento Hilal". Lo que llama la atención aquí es que Hilal haya adoptado un nombre clave de la cristiandad: Salvatus. Parece evidente que Hilal, como nombre árabe, le sirvió sin ningún problema durante los primeros dieciocho años de su abadiado. La comunidad monástica prosperó, e Hilal se enriqueció y se hizo famoso. Fueron testigos de su testamento Elvira (la reina madre y regente del infante Alfonso $\mathrm{V}$ de León, quien subió al trono en el año 999 cuando tenía cinco años), Menendo González también regente, y cinco obispos, los de Mondonedo, Lugo, Iria, Oviedo y León. Hilal legó su fortuna personal y toda su hacienda al monasterio de Valdesalce. ¿Qué ocurrió en el año 992 que le obligó a cristianizar su nombre? Vale la pena conjeturar que Hilal agregó Salvatus a su nombre en reconocimiento de una ofensa contra la madre iglesia. Según la hipótesis arriba expuesta, Hilal habría emigrado de al-Andalus a mediados del siglo $\mathrm{x}$, y se habría establecido en tierras leonesas. Eligió una carrera monástica, y, dedicado a su vocación, ascendió a abad. Por razones personales o sociales, quizás como consecuencia de un escándalo, relegó a segundo lugar su nombre árabe, pero no lo eliminó del todo. (Para otros abades en la lista podría elaborar carreras hipotéticas parecidas, aunque menos espectaculares).

Llego a una conclusión sencilla de mi planteamiento original. Los mustcaribün o mustcariba fueron individuos arabizados que vivían bajo dominio musulmán en al-Andalus, sin ninguna lealtad religiosa. Éstos emigraron al norte, a mi modo de ver para mejorar sus posiciones, y una vez allí fueron responsables de la transmisión de influjos árabes e islámicos. Por su presencia en territorios leoneses evolucionó el mozarabismo. 